\title{
Effects of Graphite and Plasticizers on the Structure of Highly Entangled Polyisoprene Melts
}

\author{
G. Giunta ${ }^{a}$, C. Svaneborg ${ }^{b}$, A. H. Karimi-Varzaneh ${ }^{c}$, P. Carbone ${ }^{a^{*}}$ \\ ${ }^{a}$ Department of Chemical Engineering and Analytical Science, The University of Manchester, Oxford Road, \\ M13 9PL, Manchester, United Kingdom \\ ${ }^{b}$ Department of Physics, Chemistry and Pharmacy, University of Southern Denmark (SDU), Campusvej 55, \\ Odense M 5230, Denmark \\ ${ }^{c}$ Continental Reifen Deutschland GmbH Rechnungsanschrift Jädekamp 30, D-30419 Hannover, Germany \\ *giuliana.giunta@manchester.ac.uk \\ "paola.carbone@manchester.ac.uk
}

\section{Supporting Information}




\section{Model and Methodology}

\section{Kremer-Grest (KG) model for the polymer bulk}

The aim of our KG model is to accurately capture the large-scale structural properties of the target polymer melts on and above the Kuhn scale. These emerge naturally from Kremer-Grest bead-and-spring models ${ }^{1}$ when the $\mathrm{KG}$ model reproduces the Kuhn Length $l_{k}$, and the dimensionless Kuhn density $n_{k}=\rho_{k} l_{k}{ }^{3}$ for the target polymer. We also identify the $\varepsilon_{K G}$ energy unit with the thermal excitation energy $k_{\mathrm{B}} T$ (where $k_{\mathrm{B}}$ is the Boltzmann constant and $T$ is the temperature) relevant for entropy dominated polymer physics

To match the dimensionless Kuhn density, we have improved the KG model with a bending potential allowing us to control chain stiffness. The reduced Kuhn density can be expressed in KG simulation parameters as $n_{k}=\rho_{b} l_{b} l_{k}^{2}$ where $\rho_{b}=0.85 \sigma^{-3}$ denotes the bead density, $l_{b}=0.965 \sigma$ the bond distance, and $l_{k}$ the Kuhn length in $\sigma$ units of the KG model. Extensive characterization of the KG model for varying chain stiffness has been performed to ascertain the dependency of the Kuhn length, entanglement length, Kuhn time and entanglement time on stiffness enabling the formulation of KG models for a wide range of target polymers. ${ }^{2}$

While the first criterion (i.e. matching the Kuhn length) allows us to design KG models with the same universal large-scale properties as any real polymer species, the second and third criteria (i.e. matching the Kuhn density and unit of energy) establish a mapping from simulation units to SI units. The Kuhn length of the KG model (in the simulation length unit $\sigma$ ) matches that of cis-PI (in SI units), fixes the size of a KG bead, $\sigma$, which in turn fixes the number of beads per monomer or Kuhn segment, and hence the molecular mass of a single bead. We note that since we retain the standard $\mathrm{KG}$ density, $\rho_{b}$, the number of beads per monomer is not a free parameter, but is determined from the two criteria.

The final parameter to fix is the unit of time. All simulations are performed at reduced temperature $T^{*}=1$. The unit of time of the KG model is defined as $\tau=\sigma\left(m_{b} \varepsilon^{-1}\right)^{0.5}$ while this unit can be expressed in SI time units using the mapping, this result is the characteristic time it takes a monomer to move its own size by ballistic motion, a time scale that has no direct relevance in polymer physics. ${ }^{2}$ Hence to obtain a meaningful time mapping we need to identify a timescale of the simulation model with an experimental time scale for instance, the entanglement time which also depends on stiffness.

Following this approach, it is possible to construct a $\mathrm{KG}$ model and relative mapping for any synthetic polymer, and this is expected, by construction, to reproduce the structural properties of the target polymer above the Kuhn scale. Since the KG model conserves the melt topology all properties such as tubes, entanglement moduli and the associated relaxation times will be reproduced. Since KG models are nearly incompressible, all the physics pertaining to the effect of correlation holes on static and dynamic properties is also expected to be reproduced by the $\mathrm{KG}$ model. Where the KG models can be expected to fail is in describing any polymer physics originating below the Kuhn scale. For instance, a KG model cannot be expected to have a meaningful glass transition temperature or hydrostatic pressure, nor will it reproduce effects 
due to hydrogen bonding or crystallization in the case of stiff polymers. Simulation studies of such properties still require the application of atomistic or coarse-grained models that has been optimized specifically to capture these specific physical effects.

Coarse-Grained Simulation of the Isoprene/Benzene Mixture

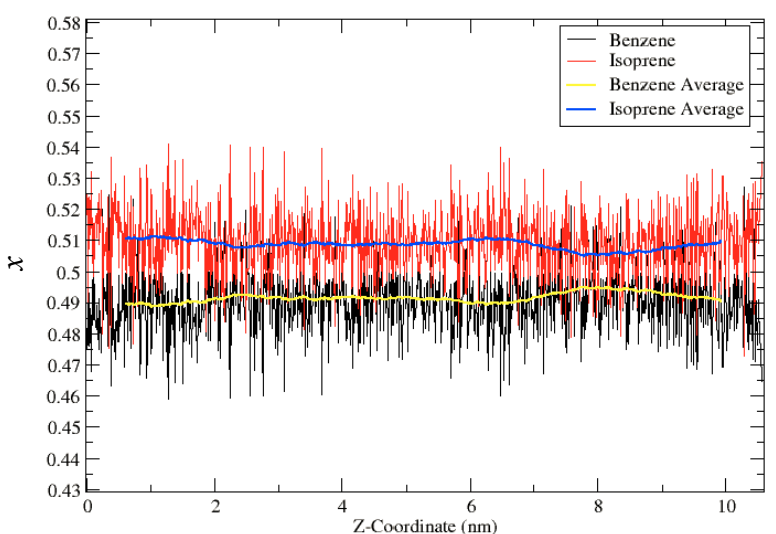

Figure S1 Profile of the mixing factor $x$ along the z-direction for the Benzene/Isoprene mixture.

List of simulations performed

Table S1 Simulated atomistic systems.

\begin{tabular}{ccc}
\hline \hline System & $\begin{array}{c}\text { Number of } \\
\text { chains }\end{array}$ & $\begin{array}{c}\text { Monomers per } \\
\text { Chain }\end{array}$ \\
\hline PI bulk & 40 & 200 \\
PI on surface & 15 & 200 \\
\hline
\end{tabular}

Table S2 Simulated CG systems.

\begin{tabular}{cccc}
\hline System & $\begin{array}{c}\text { Beads per } \\
\text { Chain }\end{array}$ & $\begin{array}{c}\text { Number of } \\
\text { Chains }\end{array}$ & $\begin{array}{c}\text { Total } \\
\text { Simulation } \\
\text { Time }\left(\boldsymbol{\tau} \times \mathbf{1 0}^{\mathbf{5}}\right)\end{array}$ \\
\hline PI bulk & 32 & 90 & 1.13 \\
PI bulk & 200 & 203 & 1.41 \\
PI on surface & 50 & 424 & 1.13 \\
PI on surface & 100 & 212 & 1.13 \\
PI on surface & 200 & 106 & 1.41 \\
PI on surface & 300 & 71 & 4.51 \\
PI on surface & 400 & 212 & 14.64 \\
PI on surface & 700 & 120 & 14.64 \\
PI on surface & 800 & 104 & 14.64 \\
\hline \hline
\end{tabular}


Table S3 Simulated Polymer/Surface systems with plasticizers.

\begin{tabular}{ccccc}
\hline \hline System & $\begin{array}{c}\text { Number of PL } \\
\text { Chains }\end{array}$ & $\begin{array}{c}\text { PL Beads per } \\
\text { Chain }\end{array}$ & $\begin{array}{c}\text { Number of PI } \\
\text { Chains }\end{array}$ & $\begin{array}{c}\text { PI Beads per } \\
\text { Chain }\end{array}$ \\
\hline PL5 & 210 & 5 & 70 & 300 \\
PL10 & 105 & 10 & 70 & 300 \\
PL20 & 53 & 20 & 70 & 300 \\
\hline \hline
\end{tabular}

Coarse-Grained Simulation of the Polymer/Surface Systems

\section{Equilibration of the PI Melt}

In order to check that the systems are relaxed with no local strains in the polymer chains, we analysed the mean square internal distance. ${ }^{3}$ At high molecular weights the fluctuations in the curves are due to statistical uncertainty in the value of the end-to-end distance.
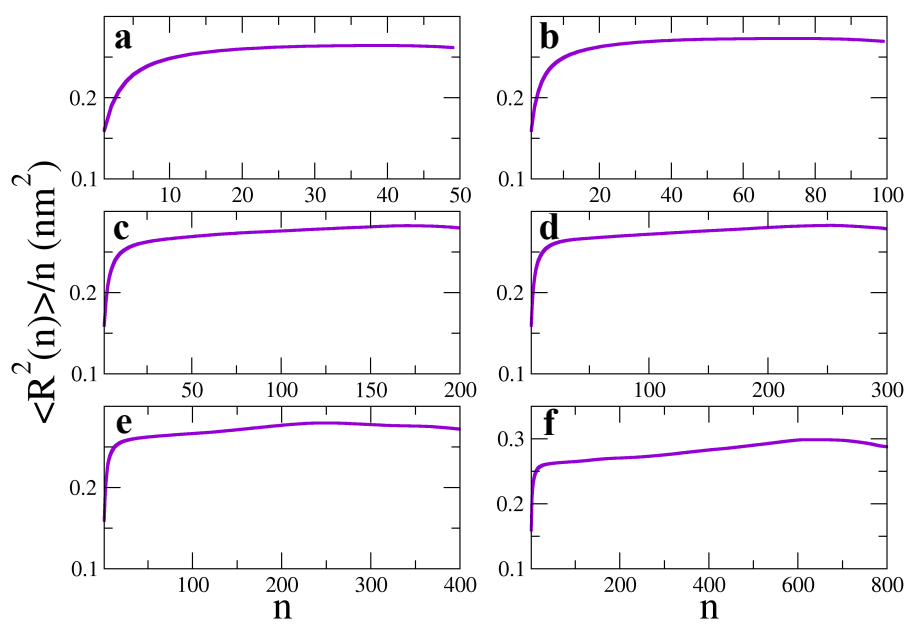

Figure S2 Mean square internal distances for different chain lengths of the KG PI chains a) 50 b) 100 c) 200 d) 300 e) 400 and f) 800 beads per chain (the maximum standard deviation of the end-to-end distance is $0.4 \mathrm{~nm}$ ). 


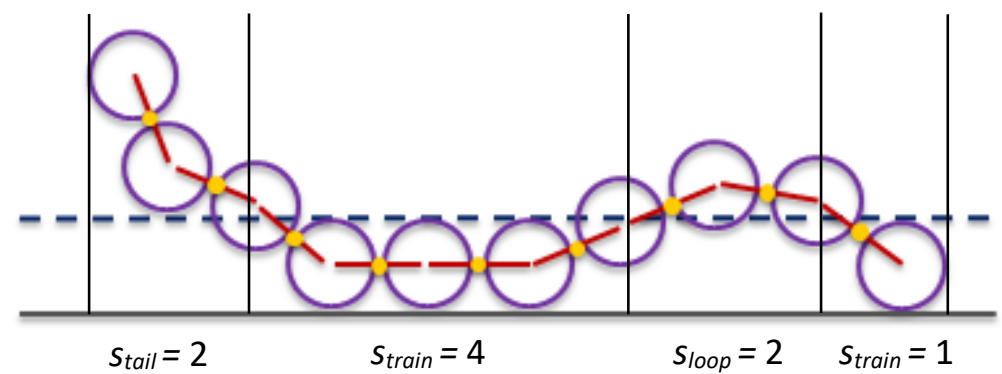

Figure S3 Schematic representation of the definitions of trains, loops and tails.
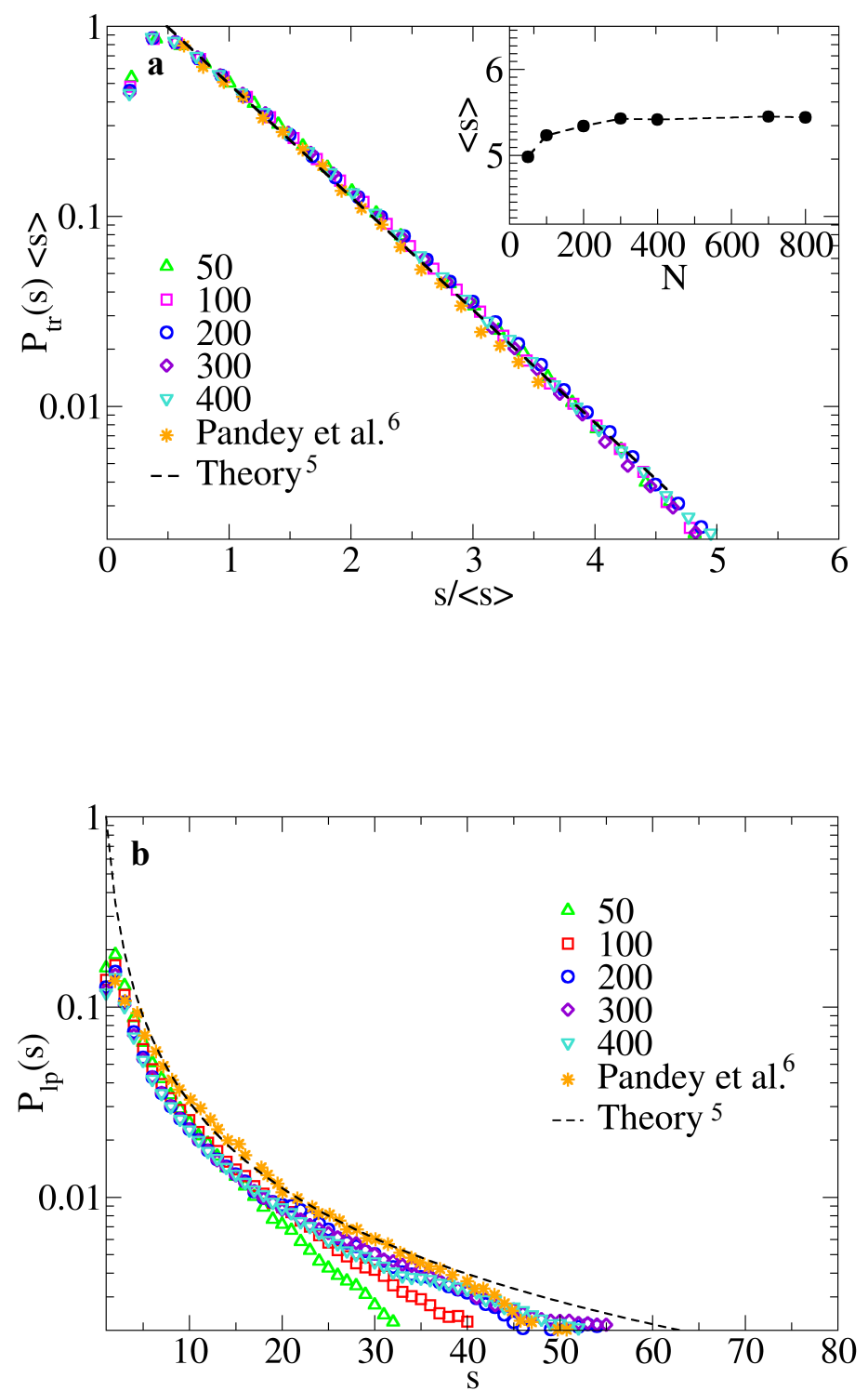


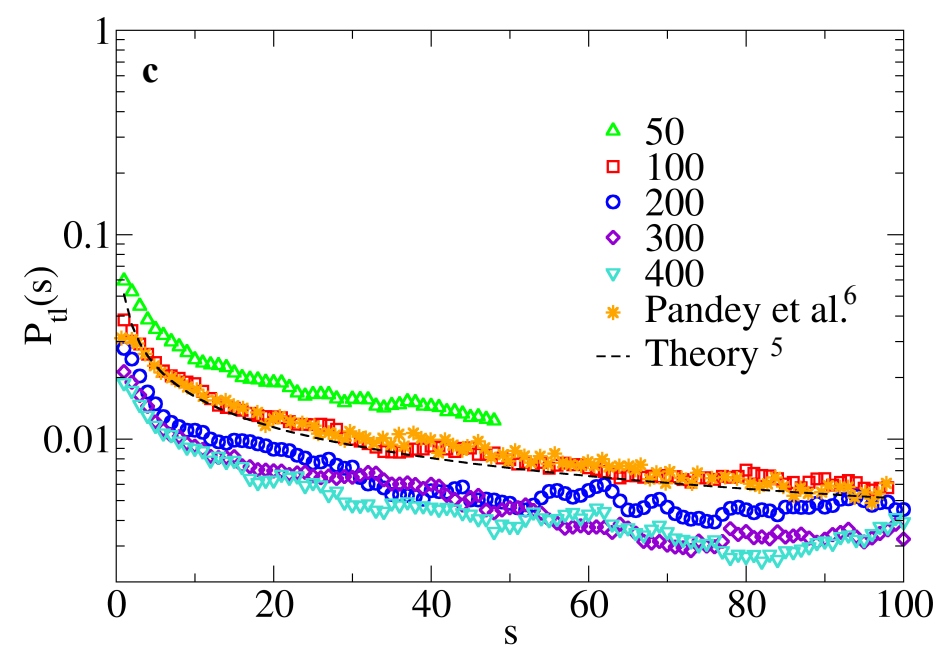

Figure S4 Distribution of: a) trains, b) loops and d) tails.

The trends of the distribution of trains and loops were compared with Hoeve's theory ${ }^{4}$ which states that the train distribution $P_{\operatorname{tr}}(s)$ follows an exponential behaviour with higher probability of short train size and it can be described using the following equation: ${ }^{5}$

$$
P_{t r}(s)=\frac{1}{<s>} e^{-\frac{s}{<s>}}
$$

where $s$ is the length of the train and $\langle s\rangle$ is the average length of the trains. As it can be observed in Figure S4 the distribution follows the trend predicted by the theory. This is an interesting result considering that the latter was developed for diluted polymer solutions where end effects were negligible.

The loop distribution is also in good agreement with the results from previous works on polymer in contact with solid surfaces. ${ }^{5-7}$ The probability distribution of loops is compared with the following equation: ${ }^{8}$

$P_{l p}(s)=\frac{1}{s^{3 / 2}} e^{-\lambda s}$

where $s$ is the length of the loop and $\lambda$ defines the adsorption free energy per monomer. Approaching the melt state $\lambda \rightarrow 0,{ }^{5}$ thus our data are compared with the function $P_{l p}(s)=$ $\frac{1}{s^{3 / 2}}$.

The tail distribution is fitted by using the equation developed by Bhattacharya et al., ${ }^{9}$ who proposed that the probability distribution was defined by:

$P_{t l}(s)=\frac{1}{s^{\beta}} e^{-\lambda s}$

where $s$ is the length tail, and $\beta=1-\gamma_{1}$, with $\gamma_{1}$ defined as the "surface exponent", which depends on the chain model: Gaussian or self-avoiding chain. Assuming that the chains in contact with the attractive surface behave as a single Gaussian chain at the critical adsorption 
point, $\gamma_{1}=1 / 2,{ }^{8}$ thus $\beta=1 / 2$ and $\lambda=0$. Therefore we fit our date with the function $P_{t l}(s) \propto s^{-1 / 2}$ as reported by Virgiliis et al. ${ }^{5}$

Furthermore, in Figure S5 we compared the train distributions for the different lattices and we observed the same trend with an average length of 5.4 and 5.7 train segments for square and hexagonal lattice, respectively. As we explain in the main text, at this CG level the lattice arrangement does not significantly affect the structure of the adsorbed chains. This approximation is valid due to the fact that we have weak interactions and the beads in the lattice are very close with uniform and smooth surface where the energy density is kept constant.

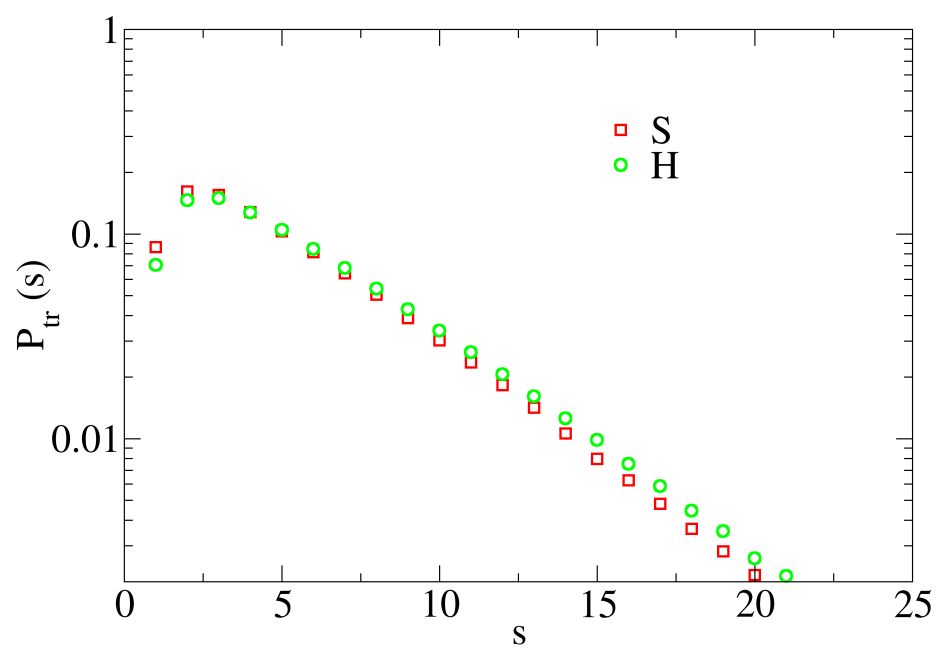

Figure S5 Comparison of the probability distribution of trains for a PI chain with 200 beads in contact with the squared lattice surface ( $\mathrm{S}$, red square) and hexagonal one (H, green circles).

Effects of the Plasticizers
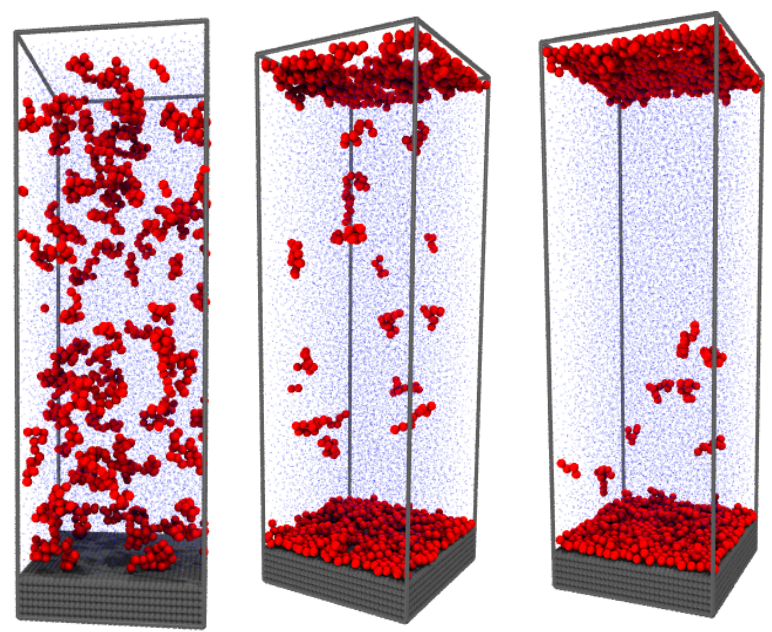

Figure S6 Equilibrated configurations of the system with PI (blue dots) and plasticizers (red) at different $\varepsilon_{p}$ values, starting from the left side $1,1.25$ and 1.5 . 


\section{Entanglement Theory}

The main observable of the PPA (Primitive Path Analysis) method is usually the chain shrinkage due to removal of thermal fluctuations. Denoting by $L_{p p}$ the average contour length of PP and $L_{c}$ the corresponding contour length of the original chains. The chain end-to-end distance is preserved by the PPA, hence we have $\langle R\rangle=a_{p p} L_{p p}=l_{K} L_{c}$ where $a_{p p}$ and $l_{K}$ denote the Kuhn length of the PP and the original chain, respectively. The number of Kuhn segments between entanglements is directly obtained as $N_{e K} \equiv a_{p p}^{2} / l_{K}^{2}=L_{p p}^{2} / L_{c}^{2}{ }^{2}$

This is a global measure of entanglements, however in the present case we are interested in entanglements close to a surface, which require a local measure. Previously, Vladkov et al. ${ }^{10}$ defined a local measure of the PP contour length, while Hoy et al. ${ }^{11}$ performed thin-chain PPA analysis, allowing them to identify and count individual entanglements and hence estimate their spatial density distribution.

Here we take a simpler more physically motivated approach to define a local entanglement measure. The mesh size $\xi$ describes the spatial distance over which chain connectivity persists in the mesh, analogously to the correlation length in a semi-dilute polymer solution. In a simple picture, if we imagine walking along a particular PP, every time we encounter an entanglement our path branches. Continuing along a random branch, we rapidly lose track of the particular PP on which we started. We can also define $\rho_{p p}$ a local mesh size as

$\xi_{p p}(z)=\left(\rho_{p p}(z)\right)^{-1 / 2}$

where $\xi_{p p}(z)$ denotes the local PP contour length density.

Volumes with a high PP contour length density chains will be strongly entangled, and hence the mesh size will be small, and we expect a small number of Kuhn segments between entanglements. For a bulk system, the PP contour length density is related to the chain number density $\rho_{c}$ as $\rho_{p p}=\rho_{c} L_{p p}$. Such that after some algebra, we can express the bulk entanglement length as $N_{e K}=\left(\rho_{K} l_{K} \xi_{p p}^{2}\right)^{2}$, where $\rho_{K}$ is the density of Kuhn segments. Using experimental values for cis-polyisoprene: $\rho_{K}=4.26 \mathrm{~nm}^{-3}, l_{K}=0.934 \mathrm{~nm}$, and $N_{e K}=24.15$ from refs 12,13 , we expect a bulk mesh size of $\xi_{p p}=1.11 \mathrm{~nm}$ or $\rho_{p p}=0.812 \mathrm{~nm}^{-2}$.

\section{References}

(1) Svaneborg, C.; Karimi-Varzaneh, H. A.; Hojdis, N.; Fleck, F.; Everaers, R. KremerGrest Models for Universal Properties of Specific Common Polymer Species. arXiv: 1606.05008 2016, 1-38.

(2) Everaers, R.; Karimi-Varzaneh, H. A.; Hojdis, N.; Fleck, F.; Svaneborg, C. KremerGrest Models for Commodity Polymer Melts: Linking Theory, Experiment and Simulation at the Kuhn Scale. 2019. Submitted for publication.

(3) Johnston, K.; Harmandaris, V. Hierarchical Multiscale Modeling of Polymer-Solid Interfaces: Atomistic to Coarse-Grained Description and Structural and Conformational Properties of Polystyrene-Gold Systems. Macromolecules 2013, 46 (14), 5741-5750. 
(4) Hoeve, C. A. J. Theory of Polymer Adsorption at Interfaces. J. Polym. Sci. Part C Polym. Symp. 1971, 34 (1), 1-10.

(5) De Virgiliis, A.; Milchev, A.; Rostiashvili, V. G.; Vilgis, T. A. Structure and Dynamics of a Polymer Melt at an Attractive Surface. Eur. Phys. J. E 2012, 35 (9), 97.

(6) Pandey, Y. N.; Brayton, A.; Burkhart, C.; Papakonstantopoulos, G. J.; Doxastakis, M. Multiscale Modeling of Polyisoprene on Graphite. J. Chem. Phys. 2014, 140 (5), 5490854913.

(7) Daoulas, K. C.; Harmandaris, V. a; Mavrantzas, V. G. Detailed Atomistic Simulation of a Polymer Melt / Solid Interface : Structure, Density, and Conformation of a Thin Film of Polyethylene Melt Adsorbed on Graphite. Macromolecules 2005, 38 (13), 57805795 .

(8) Scheutjens, J. M. H. M.; Fleer, G. J. Statistical Theory of the Adsorption of Interacting Chain Molecules. 2. Train, Loop, and Tail Size Distribution. J. Phys. Chem. 1980, 84 (2), 178-190.

(9) Bhattacharya, S.; Rostiashvili, V. G.; Milchev, A.; Vilgis, T. A. Forced-Induced Desorption of a Polymer Chain Adsorbed on an Attractive Surface: Theory and Computer Experiment. Macromolecules 2009, 42 (6), 2236-2250.

(10) Vladkov, M.; Barrat, J. L. Local Dynamics and Primitive Path Analysis for a Model Polymer Melt near a Surface. Macromolecules 2007, 40 (10), 3797-3804.

(11) Hoy, R.; Grest, G. S. Entanglements of an End-Grafted Polymer Brush in a Polymeric Matrix. Macromolecules 2007, 40 (23), 8389-8395.

(12) Fetters, L. J.; Lohse, D. J.; Colby, R. H. Chain Dimensions and Entanglement Spacings In: Mark J.E. (eds) Physical Properties of Polymers Handbook. Springer, New York, NY 2007.

(13) Svaneborg, C.; Everaers, R. Characteristic Time and Length Scales in Kremer-Grest Bead-Spring Polymer Melts as a Function of Chain Stiffness. arXiv:1808.03503 2018, $1-16$. 